\title{
Calculations of the onset temperature for tunneling in multispin systems
}

\author{
S. M. Vlasov ${ }^{1,2}$, P. F. Bessarab ${ }^{1,2}$, V. M. Uzdin ${ }^{1,3}$, H. Jónsson ${ }^{2,4}$ \\ ${ }^{1}$ ITMO University, Kronverkskiy, 49, St. Petersburg, 197101, Russia \\ ${ }^{2}$ Science Institute and Faculty of Physical Sciences, Univ. of Iceland, 107 Reykjavík, Iceland \\ ${ }^{3}$ St. Petersburg State University, St. Petersburg, 198504, Russia \\ ${ }^{4}$ Department of Applied Physics, Aalto University, Espoo, FIN-00076, Finland \\ v_uzdin@mail.ru
}

PACS 75.45. $+\mathbf{j}$, 82.20.Xr

DOI 10.17586/2220-8054-2017-8-4-454-461

\begin{abstract}
Transitions between magnetic states of a system coupled to a heat bath can occur by exceeding the energy barrier, but as temperature is lowered quantum mechanical tunneling through the barrier becomes the dominant transition mechanism. A method is presented for estimating the onset temperature for tunneling in a system with an arbitrary number of spins using the second derivatives of the energy with respect to the orientation of the magnetic vectors at the first order saddle point on the energy surface characterizing the over-the-barrier mechanism. An application to a monomer and a dimer of molecular magnets containing a $\mathrm{Mn}_{4}$ group is presented and the result found to be in excellent agreement with reported experimental measurements.
\end{abstract}

Keywords: magnetic transitions, tunneling, rate theory, molecular magnet.

Received: 5 August 2017

Revised: 15 August 2017

\section{Introduction}

An assessment of the stability of magnetic states is an important problem in the theory of magnetism $[1,2]$. Magnetic nanosystems are candidates for spintronics and high density storage devices where a critical issue is the lifetime of prepared magnetic states. A given magnetic state of a nanosystem coupled to a heat bath can have a finite lifetime because of thermally activated transitions involving leaps over an energy barrier separating it from other magnetic states of the system. But, at low enough temperature, quantum mechanical tunneling through the barrier will become the dominant transition mechanism. It is important to have a tool to estimate the temperature at which the tunneling mechanism becomes predominant, thereby reducing the lifetime from what would be expected based on the over-the-barrier mechanism.

Quantum tunneling in spin systems has been the topic of many theoretical [3] and experimental studies [4-7] over the past few decades. At high enough temperature, quantum effects are not significant and transitions are due to thermal activation described by the Arrhenius law. However, in the limit of zero temperature, only tunneling out of the lowest energy level of the initial state can occur. In an intermediate temperature range, tunneling can be thermally assisted, i.e. occurring from thermally activated levels of the initial state. The crossover from over-the-barrier to quantum tunneling can, in such cases, be relatively smooth. The shape of the energy surface affects how sharp this crossover is [8].

Several theoretical studies of the crossover in simple spin models containing one [9-12] or two [13] spins have been carried out using the effective potential method [14]. The effective potential method uses an exact mapping of a spin system onto a particle system with an effective potential and mass that can be coordinate dependent. However, this method is not general and can only be applied to simple model systems. High-order anisotropy terms, such as $\left(\hat{S}_{+}^{2}+\hat{S}_{-}^{2}\right)$ and $\left(\hat{S}_{+}^{4}+\hat{S}_{-}^{4}\right)$, have not been incorporated in the mapping approach [15], but can significantly affect the tunneling rate $[5,16]$. So far, systems with high-order anisotropy have only been studied numerically by direct diagonalization of the Hamiltonian $[17,18]$.

Here, a method for calculating the onset temperature of thermally assisted tunneling in a multispin system is presented and applied to two systems: a monomer and a dimer of molecular magnets containing $\mathrm{Mn}_{4}$ groups. The method involves an analysis of the shape of the energy surface in the vicinity of the first order saddle point on the energy surface representing the transition state of the over-the-barrier mechanism. The present article extends a previous report on systems consisting of a single magnetic vector (a single spin, or multiple spins within the macro-spin approximation) [19]. With the present formulation, the onset temperature for tunneling in systems consisting of an arbitrary number of spins can be estimated. 
The article is organized as follows: In the next section, the methodology for estimating the lifetime of a magnetic state due to over-the-barrier transitions is briefly reviewed. Then, the expression for the onset temperature for quantum mechanical tunneling is derived in section 3. Applications are presented in section 4, first to a monomer and then to a dimer of molecular magnets. The article concludes with a summary in section 5 .

\section{Over-the-barrier transitions}

A system of $N$ spins is described by the orientation of each of the spins, $\omega=\left\{\theta_{1}, \phi_{1}, \theta_{2}, \ldots \phi_{N},\right\}$, where $\theta$ and $\phi$ are spherical polar coordinates. The energy as a function of these variables, $U(\omega)$, represents an energy surface and the magnetic states of the system correspond to local minima on this surface. The mechanism of a transition from one state to another involving a jump over an energy barrier is characterized by a minimum energy path (MEP) connecting the corresponding minima. At every point on an MEP, the gradient of the energy point is along the path. The MEP is a transition path with maximal statistical weight, assuming a Boltzmann distribution is established and maintained in the initial state. The MEP can be found using the geodesic nudged elastic band method where the path is discretized by generating a set of replicas of the system and an optimization algorithm is used to bring the replicas from some initial location to an MEP [20].

The point of highest energy along the MEP, $\omega^{\dagger}$, represents a first order saddle point on the energy surface, a point where the Hessian has one and only one negative eigenvalue. Within harmonic transition state theory (HTST), the transition state is taken to be a hyperplane going through the saddle point with normal pointing along the direction of the unstable mode (the eigenvector corresponding to the negative eigenvalue of the Hessian) [21,22]. An estimate of the activation energy for the transition is then obtained as $\Delta E=U\left(\omega^{\dagger}\right)-U\left(\omega_{m}\right)$, where $U\left(\omega^{\dagger}\right)$ is the energy at the first order saddle point and $U\left(\omega_{m}\right)$ is the energy of the initial state minimum.

The rate of thermally activated transitions involving jumps over the energy barrier can be estimated using HTST for magnetic systems [21]

$$
\Gamma^{H T S T}=\nu_{0} e^{-\Delta E / k_{B} T}
$$

where $\nu_{0}$ is a pre-exponential factor that depends on the Hessian evaluated at the saddle point and at the minimum of the energy surface. Calculations of transition rates in magnetic systems using this approach have been carried out in studies of, for example, remagnetization in small islands adsorbed on a solid surface [23], analysis of temperature dependence of hysteresis loops [24], annihilation of magnetic skyrmions [25,26] and interaction of a magnetic tip with a solid surface [27].

Quantum mechanical effects can influence the transition rate even at temperature above the onset temperature for tunneling. This occurs because of the zero point energy (ZPE) which can raise the energy of the initial state and the transition state to a different extent. Within the harmonic approximation, the correction is obtained by adding the ZPE for each vibrational (magnon) mode to the initial state energy and the saddle point energy. As there is one fewer vibrational mode at the transition state than at the initial state, this effect tends to reduce the energy barrier and lower the activation energy for the transition, analogous to the so-called kinetic isotope effect in atomic rearrangements (chemical reactions and atom diffusion). The ZPE corrected activation energy is

$$
\Delta E^{Z P E C}=\left(U\left(\omega^{\dagger}\right)+\frac{1}{2} \sum_{i=1}^{N-1} \nu_{\dagger, i}\right)-\left(U\left(\omega_{m}\right)+\frac{1}{2} \sum_{i=1}^{N} \nu_{m, i}\right),
$$

where $\nu_{\dagger, i}$ and $\nu_{m, i}$ are the vibrational frequencies $[28,29]$ calculated at the saddle point and at the minimum, respectively. As can be seen from the molecular magnet example below, the ZPE correction can significantly change the acitvation energy and thereby the slope in the Arrhenius graf.

\section{Onset temperature for tunneling}

Tunneling through an energy barrier can take place because of quantum mechanical delocalization which enables the system to avoid the top of the energy barrier. In a thermalized system, the effect of delocalization can be described in a convenient way using statistical Feynman Path Integrals. The instanton technique can be used to identify when quantum delocalization becomes large enough to make tunneling the dominant transition mechanism. In instanton theory the transition rate is written in terms of the imaginary-time $(\tau=i \mathrm{t})$ action, $S$, and a stationary phase approximation [30-32]. The transition rate constant can be expressed as

$$
\Gamma=D \exp \left(-S[\omega(\tau)]_{\text {inst }} / \hbar\right)
$$

where $\omega(\tau)$ is a path corresponding to a periodic trajectory with period $\tau=\beta \hbar$ satisfying $\omega(0)=\omega(\beta \hbar)$, and the pre-factor $D$ takes into account the probability of paths deviating from $\omega(\tau)$. This special path, often referred to 
as the instanton, corresponds to a stationary point of the action, a saddle point on the action surface where the path is extended, corresponding to quantum delocalization [32-34].

In our previous work [19], we considered systems with a single spin. Here, a generalization to many-spin systems is described. The Hilbert space of a many-spin system is a tensor product of the Hilbert space of the individual spins. The Euclidean (imaginary-time) action for a system with $N$ spins of length $s$ is defined by [35-37]

$$
S(\boldsymbol{\Omega})=-i s \int_{-\beta / 2}^{\beta / 2} \sum_{j=1}^{N}\left(1-\cos \theta_{j}\right) \dot{\phi}_{j} d \tau+\int_{-\beta / 2}^{\beta / 2} U(\omega(\tau)) d \tau,
$$

where the first term is the sum of the Berry phase of the individual spins and $\Omega=\omega(\tau)$ represents a closed trajectory with period $\beta$.

From here on we use atomic units with $\hbar=1, \mu_{B}=1 / 2$ and the mass and charge of an electron have unit value, $e=1$ and $m_{e}=1$. A scaled gyromagnetic ratio is defined as $\tilde{g}=g / 2$.

In order to find the onset temperature for tunneling, $T_{c}$, the action is expanded to second order in the vicinity of the first order saddle point on the energy surface, $\boldsymbol{\Omega}=\omega^{\dagger}+\epsilon \delta \boldsymbol{\Omega}$,

$$
\begin{gathered}
S(\boldsymbol{\Omega})=\beta U\left(\omega^{\dagger}\right)+\delta S+\frac{1}{2} \delta^{2} S, \\
\delta^{2} S=-2 i s \int_{-\beta / 2}^{\beta / 2} \sum_{j=1}^{N}\left(\delta \theta_{j} \delta \dot{\phi}_{j} \sin \theta_{j}\right) d \tau+\int_{-\beta / 2}^{\beta / 2} \delta \boldsymbol{\Omega} \mathcal{H} \delta \boldsymbol{\Omega}^{T} d \tau,
\end{gathered}
$$

where $\mathcal{H}$ is the Hessian matrix of the energy surface $U(\omega)$ at the saddle point $\omega^{\dagger}$. As $\epsilon \rightarrow 0, \delta^{2} S$ becomes a quadratic form of the Hessian, $\mathcal{H}$, which has one and only one real negative eigenvalue.

The task is to find the temperature at which $\Omega$ becomes an instanton, i.e. a saddle point at the action surface with quantum delocalization. At that point, a zero mode appears corresponding to displacement along the path and thus constant $S$. This signals the transition from thermally activated jumps to quantum tunneling $[32,33]$.

Since the instanton is a closed path, $\delta \theta_{k}$ and $\delta \phi_{k}$ can be expanded in a Fourier series and $\delta^{2} S$ in eqn. (6) rewritten as

$$
\begin{array}{r}
\frac{1}{2} \delta^{2} S\left(\theta^{\dagger}, \phi^{\dagger}\right)=\beta \sum_{n=0}^{\infty}\left(\sum_{k=0}^{N}\left[\frac{2 \pi s \sin \theta_{k}^{\dagger}}{\beta} n\left(\phi_{n}^{k} \theta_{n}^{k^{*}}-\phi_{n}^{k^{*}} \theta_{n}^{k}\right)\right]+\right. \\
\left.\sum_{j, k}\left[a \theta_{n}^{k} \theta_{n}^{j^{*}}+b\left(\phi_{n}^{k} \theta_{n}^{j^{*}}+\phi_{n}^{k^{*}} \theta_{n}^{j}\right)+c \phi_{n}^{k} \phi_{n}^{j^{*}}\right]\right)
\end{array}
$$

The matrix representing the quadratic form of the action is infinite and has a block diagonal form

$$
G=\left(\begin{array}{ccccc}
\gamma_{0} & & & & \\
& \gamma_{1} & & & \\
& & \ddots & & \\
& & & \gamma_{n} & \\
& & & & \ddots
\end{array}\right)
$$

where

$$
\gamma_{n}=\mathcal{H}+\left(\begin{array}{ccc|ccc}
0 & \cdots & 0 & -k_{1}^{n} & \cdots & 0 \\
0 & \ddots & 0 & 0 & \ddots & 0 \\
0 & \cdots & 0 & 0 & \cdots & -k_{N}^{n} \\
\hline k_{1}^{n} & \cdots & 0 & 0 & \cdots & 0 \\
0 & \ddots & 0 & 0 & \ddots & 0 \\
0 & \cdots & k_{N}^{n} & 0 & \cdots & 0
\end{array}\right)
$$

where $k_{j}^{n}=2 \pi n T s \sin \theta_{j}^{\dagger}$.

An expression for the onset temperature for tunneling can be obtained by analyzing the determinant of $G$ :

$$
\operatorname{det}(G)=\prod_{n=0}^{\infty} \operatorname{det}\left(\gamma_{n}\right)
$$


Consider first $\gamma_{0}$ which is the Hessian of the energy surface at the first order saddle point. There, $\gamma_{0}$ contains one negative eigenvalue so $\operatorname{det}\left(\gamma_{0}\right)<0$. However, the other blocks, $\gamma_{n}$ (for $n>0$ ), depend on temperature and at $T \gg T_{c}$ are positive definite

$$
\operatorname{det}\left(\gamma_{n}\right) \sim(2 \pi s n)^{2 N} \prod_{j=1}^{N} \sin ^{2} \theta_{j}^{\dagger}>0
$$

and $\operatorname{det}(G)<0$. As the temperature is lowered to $T_{c}$, a zero of $G$ appears signaling a stationary point involving quantum delocalization. This must arise from $\gamma_{1}$ since the temperature in each $\gamma_{n}$ is scaled by $n$ and blocks with $n>1$ thus have zeros at even lower temperature. The onset temperature for tunneling is, therefore, found by solving

$$
\operatorname{det}\left(\gamma_{1}\left(T_{c}\right)\right)=0
$$

This can be easily done numerically for multi-spin systems. All that is required for the calculation are the second derivatives of the energy at the first order saddle point corresponding to the over-the-barrier mechanism.

For a single-spin system, an analytical expression for the onset temperature can be obtained

$$
T_{c}=\frac{\sqrt{b^{2}-a c}}{2 \pi s k_{B} \sin \theta^{\dagger}}
$$

where

$$
\left.a \equiv \frac{\partial^{2} U\left(\theta^{\dagger}, \phi^{\dagger}\right)}{\partial \theta^{2}}\right|_{\theta^{\dagger}, \phi^{\dagger}},\left.c \equiv \frac{\partial^{2} U\left(\theta^{\dagger}, \phi^{\dagger}\right)}{\partial \phi^{2}}\right|_{\theta^{\dagger}, \phi^{\dagger}},\left.b \equiv \frac{\partial^{2} U\left(\theta^{\dagger}, \phi^{\dagger}\right)}{\partial \theta \partial \phi}\right|_{\theta^{\dagger}, \phi^{\dagger}} .
$$

The pair of eigenvalues of the $\gamma_{1}$ block are

$$
\lambda=\frac{a+c}{2} \pm \frac{\sqrt{(a-c)^{2}+4 b^{2}-4 k^{2}}}{2} .
$$

In addition to the crossover temperature, we can define the temperature $T_{0}$ at which the two eigenvalues become complex conjugates

$$
T_{0}=\frac{\sqrt{(a-c)^{2}+4 b^{2}}}{4 \pi s k_{B} \sin \theta^{\dagger}} .
$$

At this temperature, both eigenvalues are equal to $(a+c) / 2$ and could be negative. At higher temperature, the eigenvalues are complex. In the temperature range between $T_{c}$ and $T_{0}$ the two eigenvalues are real, either both are positive or both are negative.

\section{Applications. Molecular magnets}

Previously, we have presented calculations and compared with experimental results for the single molecular magnet containing a $\mathrm{Mn}_{4}$ group [19]. We briefly review those calculations here before discussing the dimer. The molecular magnet has a total spin of $s=9 / 2$ and can be described with the following Hamiltonian

$$
\hat{H}=-D \hat{S}_{z}^{2}-B \hat{S}_{z}^{4}-g \mu_{0} \hat{\mathbf{S}} \cdot \mathbf{H}+\hat{H}_{\text {trans }}
$$

where $D$ and $B$ are axial anisotropy constants, $\mathrm{B}$ is an applied magnetic field and $H_{\text {trans }}$ gives the in-plane anisotropy which is small compared with $D$. The values of the parameters have been determined from experimental measurements (not involving tunneling) [38]: $D=0.74 \mathrm{~K}, B=-3.8 \mathrm{mK}$ and $g=2$. The transverse term is chosen to be fourth-order $E\left(\hat{S}_{+}^{4}+\hat{S}_{-}^{4}\right.$ with $E=9.8 \times 10^{-4} \mathrm{~K}[38]$.

For the single molecular magnet it is possible to give an analytical formula for the onset temperature. Expanding (12) with the $\mathrm{Mn}_{4}$ system Hamiltonian (17) at the saddle point gives

$$
T_{c}=\frac{4 s^{2} \sqrt{D E-4 E^{2} s^{2}}}{\pi}
$$

and $T_{c}=0.61 \mathrm{~K}$. This result is in close agreement with the reported experimental results giving $0.67 \mathrm{~K}$ [38]. The dependence of eigenvalues on temperature is shown in Fig. 1.

The calculated transition rate using HTST (applicable for $T>T_{c}$ ) and the predicted onset temperature for tunneling in the monomer are shown in Fig. 2, along with experimental data. At temperature below $T_{c}$, the tunneling rate is expected to be similar to the HTST rate at $T_{c}$. The agreement between the calculations and measurements is good. 


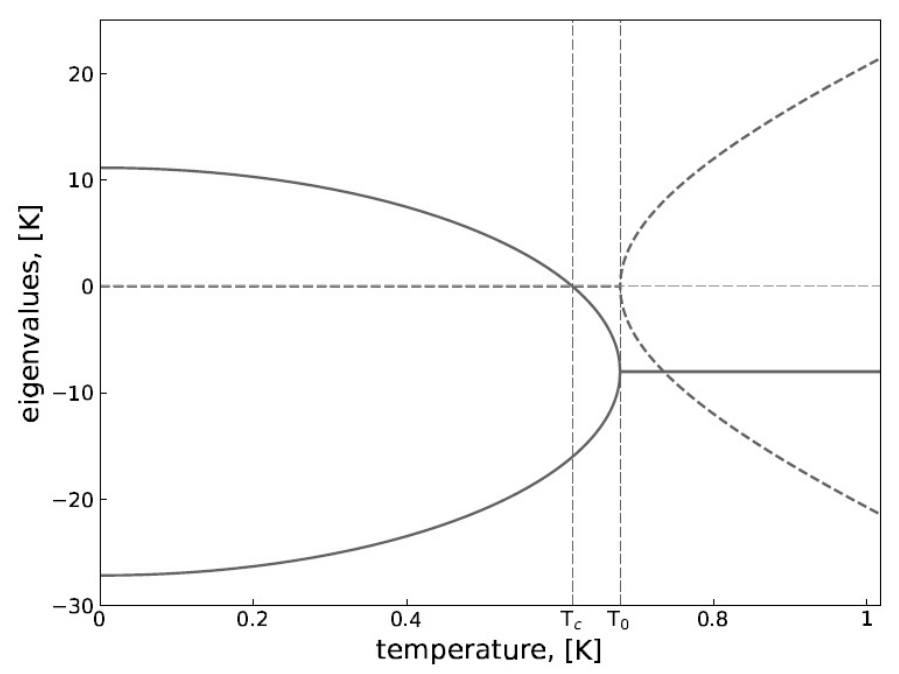

FIG. 1. Eigenvalues of the $\gamma_{1}$ matrix block for the monomer (eq. (17)) $\mathrm{Mn}_{4}$ molecular magnet. Solid line: real part. Dashed line: imaginary part. At temperature above $T_{0}$ the eigenvalues are complex conjugates and give a positive contribution into the determinant. At temperature below the $T_{c}, \gamma_{1}$ has only one negative eigenvalue, therefore the determinant changes sign at $T_{c}$. In the temperature interval $T_{c}<T<T_{0} \gamma_{1}$ has two negative eigenvalues

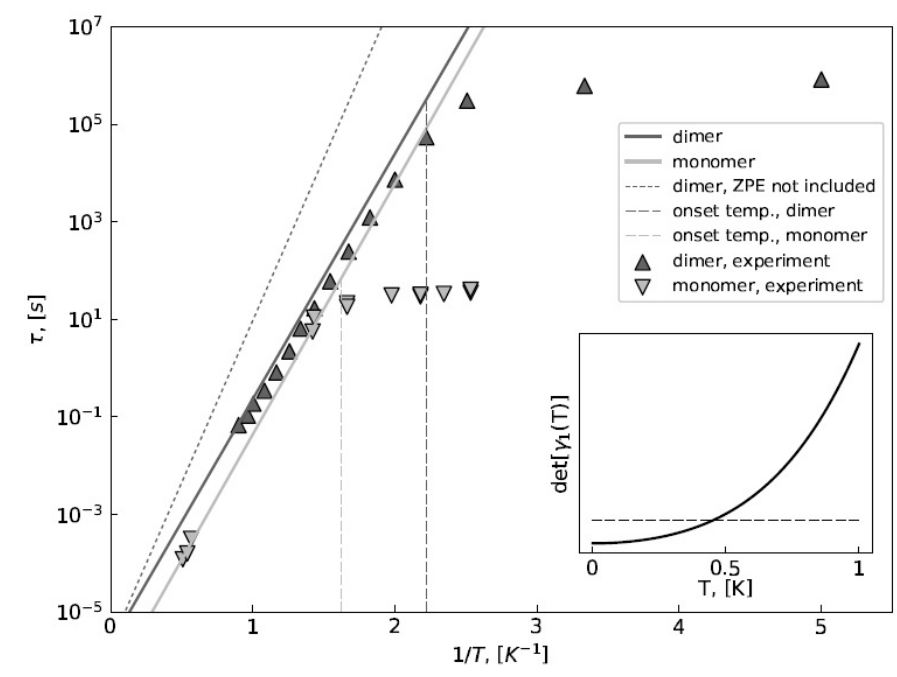

FIG. 2. The calculated lifetime for a monomer and a dimer of molecular magnets containing $\mathrm{Mn}_{4}$ groups. Results of calculations using harmonic transition state theory with zero point energy correction for the monomer (solid gray line) and the dimer (solid black line) are shown. Calculated lifetime for the dimer without ZPE correction (dashed line) is also shown for comparison. Calculated values for the onset temperature for tunneling calculated from (18) using the Hamiltonian in (17) for the monomer and (19) for the dimer (dashed vertical lines) with parameters taken from experimental measurements are also shown. Experimentally measured transition rate for the monomer [38] is shown with gray triangles and for the dimer [40] with black triangles. Below $T_{c}$, the tunneling rate is expected to be similar to the HTST rate at $T_{c}$. Excellent agreement is obtained between the calculated and measured results. Inset: The determinant of the $\gamma_{1}$ matrix block for the dimer, showing how it becomes negative at $T_{c}$ 
We turn now to calculations for a dimer of $\mathrm{Mn}_{4}$ molecular magnets and compare with experimental measurements on $\left[\mathrm{Mn}_{4} \mathrm{O}_{3} \mathrm{Cl}_{4}\left(\mathrm{O}_{2} \mathrm{CEt}\right)_{3}(\mathrm{py})_{3}\right]_{2}$. Each unit of the dimer is described with a magnetic moment of $s=9 / 2$, and the Hamiltonian of the system is taken to be

$$
\hat{H}=\hat{H}_{1}+\hat{H}_{2}+J \hat{\mathbf{S}}_{1} \cdot \hat{\mathbf{S}}_{2}
$$

where both $\hat{H}_{1}$ and $\hat{H}_{2}$ have the same form as (17) with the parameters taken from [39] and $J=0.12 \mathrm{~K}$ is isotropic superexchange [40]. The transverse term is chosen to include both 4th- and 2nd-order anisotropies: $C\left(\hat{S}_{+}^{2}+\hat{S}_{-}^{2}\right)$ and $E\left(\hat{S}_{+}^{4}+\hat{S}_{-}^{4}\right)$, with $C=0.032 \mathrm{~K}$ and $E=-7.5 \times 10^{-5} \mathrm{~K}$, in order to match the experimental results of the rate above $T_{c}$. The energy surface and a minimum energy path for a transition are shown in Fig. 3.

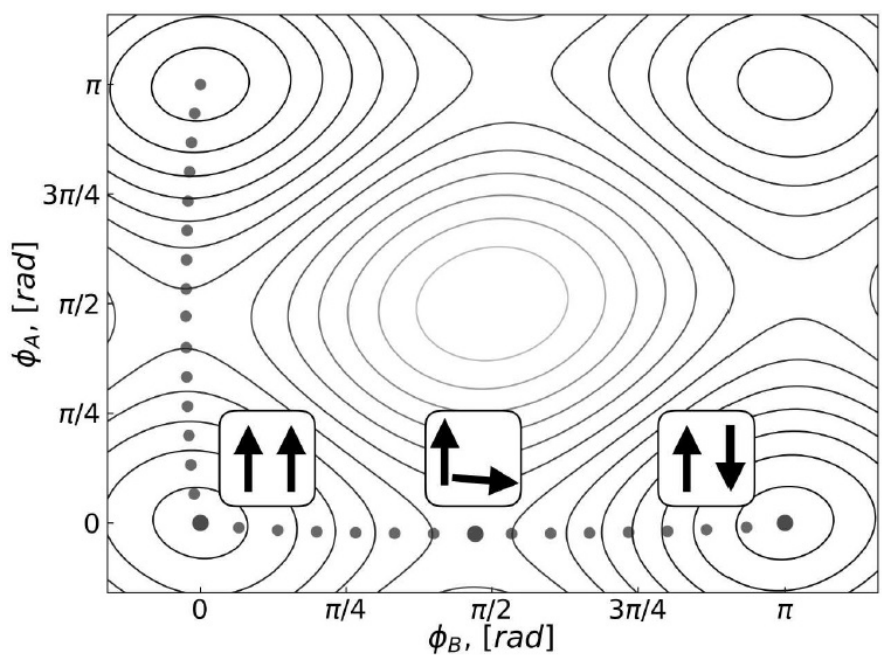

FIG. 3. Energy surface for a dimer molecular magnets containing $\mathrm{Mn}_{4}$ group when $\theta_{A}=\theta_{B}=$ $\pi / 2$. The minimum energy path for a remagnetization transition is shown with black dots. The insets show the orientation of the $9 / 2$ spins of each $\mathrm{Mn}_{4}$ unit at the initial state (left), first order saddle point (middle), and product state (right).

The calculated crossover temperature for the dimer is $T_{c}=0.40 \mathrm{~K}$ in close agreement with experimental measurements [40]. Due to the weak exchange interaction every spin flips almost independently.

In order to see the effect of coupling between the two units more clearly, we also calculated and analyzed a system with larger exchange interaction, taken to be of the same order as the anisotropy, $J=0.6 \mathrm{~K}$. The calculated onset temperature for the tunneling is found to be $T_{c}=0.35 \mathrm{~K}$. Due to the strong exchange interaction between spins eigenvalues of $\gamma_{1}$ behave differently, but we still can see the temperature at which one of them crosses zero (see Fig. 4).

The result of calculation of the HTST transition rate and onset temperature for tunneling of the dimer (19) using experimentally determined parameter values is shown in Fig. 2. The ZPE correction changes the slope in the Arrhenius graph, bringing the calculated HTST results closer to the experimental values.

\section{Summary}

A method is presented for finding the onset temperature of thermally activated tunneling in magnetic systems that are characterized by spin vectors with orientation prescribed by continuous angular variables. A study is made for the $\mathrm{Mn}_{4}$ molecular magnets where both the high temperature jump rate and the onset temperature for tunneling are calculated and compared with experimental data. Excellent agreement is obtained. The rate calculated above the onset temperature for tunneling includes zero point energy correction. The onset temperature for tunneling is found by analyzing eigenvalues of the $\gamma_{1}$ matrix block at the first order saddle point on the energy surface.

Previously, the onset temperature for tunneling in single-spin or simple (without higher-order anisotropy) twospin systems can be assessed by other methods, such as the effective potential method or numerical methods involving direct diagonalization of the Hamiltonian, but the method presented here is applicable to systems with an arbitrary number of spins. The method presented here, makes it possible to estimate the onset temperature for tunneling in a magnetic system described by spin vectors as long as the second derivatives of the energy with respect to the angles describing the orientation of vectors can be evaluated at the saddle point on the energy surface. 


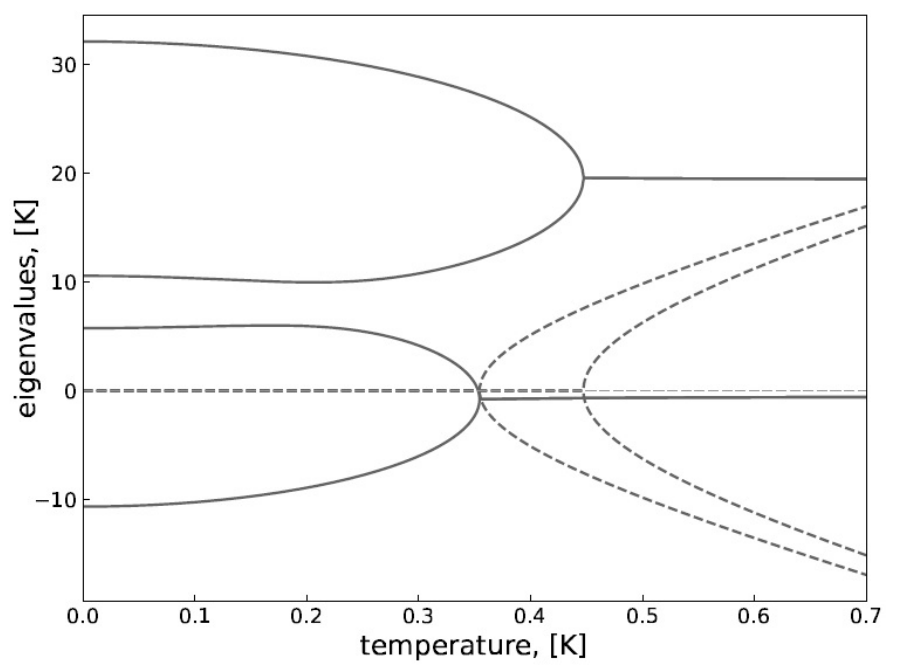

FIG. 4. Eigenvalues of the $\gamma_{1}$ matrix block of the dimer (eq. (19)) when the exchange parameter is chosen to be $J=0.6 \mathrm{~K}$. Solid line: real part. Dashed line: imaginary part. The eigenvalues depend on temperature in a similar way as is shown for the monomer in Fig. 1. A pair of eigenvalues becomes real and remains positive, while a second pair of eigenvalues changes sign at $T_{c}$ from negative to positive as temperature is lowered

The most peculiar characteristic of magnetic systems is that the onset temperature for tunneling depends not only on second derivatives along the unstable mode at the first order saddle point, as in particle systems, but on the curvature of the energy surface at the first order saddle point. This is due to the inseparability of the spin Hamiltonian.

\section{Acknowledgements}

This work was supported by the Icelandic Research Fund and the Academy of Finland (grant 278260) and partially financially supported by the Government of the Russian Federation (grant 074-U01).

\section{References}

[1] Brown, Jr. W.F. Thermal Fluctuations of Fine Ferromagnetic Particles. IEEE Transactions on Magnetics, 1979 , MAG-15, P. 1196-1208.

[2] Braun H.-B. Kramers's rate theory, broken symmetries and magnetization reversal. J. Appl. Physics, 1994, 76, P. 6310-6315.

[3] Chudnovsky E.M., Tejada J. Macroscopic Quantum Tunneling of the Magnetic Moment. Cambridge University Press, New York.: 1998.

[4] Bokacheva L., Kent A.D. and Walters M.A. Crossover between Thermally Assisted and Pure Quantum Tunneling in Molecular Magnet $M n_{12}$-Acetate. Phys. Rev. Lett., 2000, 85, P. 4803-4806.

[5] Mertes K.M., Zhong Y., et al. Abrupt crossover between thermally activated relaxation and quantum tunneling in a molecular magnet. Europhys. Lett., 2001, 55, P. 874-879.

[6] Wernsdorfer W., Murugesu M. and Christou G. Resonant Tunneling in Truly Axial Symmetry $M n_{12}$ Single-Molecule Magnets: Sharp Crossover between Thermally Assisted and Pure Quantum Tunneling. Phys. Rev. Lett., 2006, 96, 057208 (4 pp.)

[7] Loth S., Baumann S., et al. Bistability in atomic-scale antiferromagnets. Science, 2012, 335, P. 196-199.

[8] Chudnovsky E.M. Phase transitions in the problem of the decay of a metastable state. Phys. Rev. A, 1992, 46, P. 8011-8014.

[9] Chudnovsky E. M., Garanin D. A. First- and Second-Order Transitions between Quantum and Classical Regimes for the Escape Rate of a Spin System. Phys. Rev. Lett., 1997, 79, P. 4469-4472.

[10] Park C.-S., Yoo S.-K. and Yoon D.-H. Quantum-classical crossover of the escape rate in a biaxial spin system with an arbitrarily directed magnetic field. Phys. Rev. B, 2000, 61, P. 11618-11624.

[11] Kim G.-H. and Chudnovsky E.M. Escape-rate crossover between quantum and classical regimes in molecular magnets: A diagonalization approach. Europhys. Lett., 2000, 52, P. 681-687.

[12] Garanin D.A. and Chudnovsky E.M. Quantum statistical metastability for a finite spin. Phys. Rev. B, 2000, 63, 024418 (7 pp.)

[13] Owerre S. A. and Paranjape M. B. Phase transition between quantum and classical regimes for the escape rate of dimeric molecular nanomagnets in a staggered magnetic field. Phys. Lett. A, 2014, 378, P. 1407-1412.

[14] Ulyanov V.V. and Zaslavskii O.B. New methods in the theory of quantum spin systems. Phys. Rep., 1992, 216, P. 179-251.

[15] Owerre S. A., Paranjape M. B. Macroscopic quantum tunneling and quantum-classical phase transitions of the escape rate in large spin systems. Phys. Rep., 2015, 546, P. 1-60.

[16] Wernsdorfer W. and Sessoli R. Quantum Phase Interference and Parity Effects in Magnetic Molecular Clusters. Science, 1999, 284, P. $133-135$.

[17] Kang D.H. and Kim G.-H. Theoretical study of abrupt or gradual crossover of the escape rate in single-molecule magnets. Phys. Rev. B, 2006, 74, 184418 (5 pp.) 
[18] Kim G.-H., Kang D.H. and Shin M.C. Quantum-classical crossover of the escape rate in the biaxial nanomagnets with a higher order symmetry. Eur. Phys. J. B, 2011, 83, P. 63-67.

[19] Vlasov S., Bessarab P.F., Uzdin V.M. and Jónsson H. Classical to quantum mechanical tunneling mechanism crossover in thermal transitions between magnetic states. Faraday Discuss., 2016, 195, P. 93-109.

[20] Bessarab P.F., Uzdin V.M. and Jónsson H. Method for finding mechanism and activation energy of magnetic transitions, applied to skyrmion and antivortex annihilation. Comp. Phys. Commun., 2015, 196, P. 335-347.

[21] Bessarab P.F., Uzdin V.M. and Jónsson H. Harmonic Transition State Theory of Thermal Spin Transitions. Phys. Rev. B, 2012, 85, 184409 (4 pp.)

[22] Bessarab P.F., Uzdin V.M. and Jónsson H. Potential Energy Surfaces and Rates of Spin Transitions. Z. Phys. Chem., 2013, 227, P. 15431557

[23] Bessarab P.F., Uzdin V.M. and Jónsson H. Size and Shape Dependence of Thermal Spin Transitions in Nanoislands. Phys. Rev. Lett., 2013, 110, 020604 (5 pp.)

[24] Moskalenko M. , Bessarab P.F., Uzdin V.M. and Jónsson H. Qualitative Insight and Quantitative Analysis of the Effect of Temperature on the Coercivity of a Magnetic System. AIP Advances, 2016, 6, 025213 (8 pp.)

[25] Lobanov I., Jónsson H. and Uzdin V. M. Mechanism and activation energy of magnetic skyrmion annihilation obtained from minimum energy path calculations. Phys. Rev. B, 2016, 94, 174418 (7 pp.)

[26] Bessarab P.F. Comment on "Path to collapse for an isolated Néel skyrmion". Phys. Rev. B, 95, 2017, 136401 (2 pp.)

[27] Ivanov A. , Bessarab P.F., Uzdin V.M. and Jónsson H. Magnetic exchange force microscopy: Theoretical analysis of induced magnetization reversals. Nanoscale, 2017, Accepted Manuscript, DOI: 10.1039/C7NR04036A

[28] Smit J., Beljers H. Ferromagnetic resonance absorption in $\mathrm{BaFe}_{12} \mathrm{O}_{19}$, a highly anisotropic crystal. Philips Res. Rep., 1955, 10, P. 113-130.

[29] Suhl H. Ferromagnetic resonance in nickel ferrite between one and two kilomegacycles. Phys. Rev., 1955, 97, P. 555-557.

[30] Miller W.H. Semiclassical limit of quantum mechanical transition state theory for nonseparable systems. J. Chem. Phys., 1975, 62, P. $1899-1906$.

[31] Callen C., Coleman S. Fate of the false vacuum. II. First quantum corrections. Phys. Rev. D, 1977, 16, P. 1762-1768

[32] Benderskii V. A., Goldanskii V. I. and Makarov D. E. Quantum dynamics in low-temperature chemistry. Phys. Rep., 1993, 233, P. 195-339.

[33] Mills G., Schenter G. K., Makarov D. E. and Jónsson H. Generalized path integral based quantum transition state theory. Chem. Phys. Letters, 1997, 278, P. 91-96.

[34] Mills G., Schenter G. K., Makarov D. E. and Jónsson H. RAW Quantum transition state theory. proceeding of "Classical and Quantum Dynamics in Condensed Phase Simulations", Villa Marigola, Lerici, Italy, 1997, 405 pp.

[35] KIauder J. Path integrals and stationary-phase approximations. Phys. Rev. D, 1979, 19, P. 2349-2356.

[36] Kochetov E. SU(2) coherentstate path integral. J. Math. Phys., 1995, 36, P. 4667-4679.

[37] Fradkin E. Field Theories of Condensed Matter Physics. Cambridge University Press, New York.: 2013, 190 pp.

[38] Aubin S. M. J. et. al. Resonant Magnetization Tunneling in the Trigonal Pyramidal $\mathrm{Mn}^{I V} \mathrm{Mn}_{3}^{I I I}$ Complex $\left[\mathrm{Mn}_{4} \mathrm{O}_{3} \mathrm{Cl}_{(}\left(\mathrm{O}_{2} \mathrm{CCH}_{3}\right)_{3}(\mathrm{dbm})_{3}\right]$. J. Am. Chem. Soc., 1998, 120, P. 4991-5004.

[39] Edwards R. S., Hill S., et. al. A comparative high frequency EPR study of monomeric and dimeric $\mathrm{Mn}_{4}$ single-molecule magnets, Polyhedron, 2003, 22, P. 1911-1916.

[40] Wernsdorfer W., Aliaga-Alcalde N., Hendrickson D. N., Christou G. Exchange-biased quantum tunnelling in a supramolecular dimer of single-molecule magnets. Nature, 2002, 416, P. 406-409. 